\title{
Randomized Protocols for Asynchronous Consensus
}

\author{
James Aspnes*
}

May 28, 2018

\begin{abstract}
The famous Fischer, Lynch, and Paterson impossibility proof shows that it is impossible to solve the consensus problem in a natural model of an asynchronous distributed system if even a single process can fail. Since its publication, two decades of work on fault-tolerant asynchronous consensus algorithms have evaded this impossibility result by using extended models that provide (a) randomization, (b) additional timing assumptions, (c) failure detectors, or (d) stronger synchronization mechanisms than are available in the basic model. Concentrating on the first of these approaches, we illustrate the history and structure of randomized asynchronous consensus protocols by giving detailed descriptions of several such protocols.
\end{abstract}

\section{Introduction}

The consensus problem [45] is to get a group of $n$ processes in a distributed system to agree on a value. A consensus protocol is an algorithm that produces such an agreement. Each process in a consensus protocol has, as part of its initial state, an input from some specified range, and must eventually decide on some output from the same range. Deciding on an output is irrevocable; though a process that has decided may continue to participate in the protocol, it cannot change its decision value. The restricted problem in which the input range is $\{0,1\}$ is called binary consensus. Except as otherwise noted, all of the protocols discussed hereafter are binary consensus protocols.

Correct consensus protocols must satisfy the following three conditions:

${ }^{*}$ Department of Computer Science, Yale University, New Haven, CT 06520-8285, USA. Email: aspnes-james@cs.yale.edu. Supported by NSF grants CCR-9820888 and CCR0098078. 
1. Agreement. All processes that decide choose the same value.

2. Termination. All non-faulty processes eventually decide.

3. Validity. The common output value is an input value of some process.

This is not precisely the definition originally given by Pease, Shostak, and Lamport [45]. Their paper used the even stronger condition of interactive consistency, in which all non-faulty processes compute the same vector of purported inputs, and this vector correctly identifies the input of each nonfaulty process. But the definition above is the one that is generally accepted today. It derives from the three-part definition used by Fischer, Lynch, and Paterson [37], though in their paper the validity condition is replaced by a much weaker non-triviality condition. Non-triviality says only that there exist different executions of the protocol that produce different common decision values. Non-triviality is implied by validity (consider an execution in which all processes have input 0 versus one in which all process have input 1), but it is less useful for applications, since it says nothing about the relationship between inputs and outputs. Nonetheless, non-triviality is enough to show that consensus is impossible in the usual model.

The Fischer-Lynch-Paterson (FLP) impossibility result [37] demonstrates that there is no deterministic protocol that satisfies the agreement, termination, and non-triviality conditions for an asynchronous messagepassing system in which any single process can fail undetectably. A similar result, proved by Loui and Abu-Amara [44] using essentially the same technique, shows that consensus is also impossible in an asynchronous sharedmemory system with at least one undetectable failure. More details of these and similar results, and of the models in which they apply, can be found in the survey by Fich and Ruppert appearing elsewhere in this volume.

And yet we would like to be able to solve consensus. To escape from the FLP result, we must extend the model in some way. Several such extensions that have been used to solve asynchronous consensus are described in Section 2. In this paper, we concentrate on the use of randomized algorithms, and give a more detailed description of randomized approaches in Sections 3 through 6 .

\section{Extensions to the model}

The extensions to the base model that have been used to circumvent the FLP result can be divided into four classes. 
1. Randomization. Randomized models provide probabilities for some transitions. This means that instead of looking at a single worstcase execution, one must consider a probability distribution over bad executions. If the termination requirement is weakened to require termination only with probability 1, the FLP argument no longer forbids consensus: non-terminating executions continue to exist, but they may collectively occur only with probability 0 .

There are two ways that randomness can be brought into the model. One is to assume that the model itself is randomized; instead of allowing arbitrary applicable operations to occur in each state, particular operations only occur with some probability. Such a randomized scheduling approach was first proposed by Bracha and Toueg [23] (who called their version fair scheduling). A recent attempt to revive this approach can be found in the noisy scheduling model of [11]. Randomized scheduling allows for very simple algorithms; unfortunately, it depends on assumptions about the behavior of the world that may not be justified in practice. Thus it has not been as popular as the second approach, in which randomness is located in the processes themselves.

In this randomized algorithm approach, processes are equipped with coin-flip operations that return random values according to some specified probability distribution. Again, we can no longer talk about a single worst-case execution, but must define a probability distribution on executions. Defining this distribution requires nailing down all the other nondeterminism in the system (i.e., the order in which messages are sent and delivered, or in which shared-memory operations are performed), which is done formally by specifying an adversary. An adversary is a function from partial executions to operations that says which applicable operation to carry out at each step. (Details are given in Section 4.) Given an adversary, the result of the coin-flip operations are the only remaining unknowns in determining which execution occurs. So we can assign to each set of executions the probability of the set of sequences of coin-flip outcomes that generate them.

The adversary we have just described is the strong adversary; it can observe the entire history of the system, including past coin-flip outcomes and the states of processes and the communications mechanism, but it cannot predict future coin-flip outcomes. A strong adversary gives a weak model in which consensus is possible but difficult. Weakening the adversary gives a stronger model (in the sense of granting 
more strength to the processes); many consensus protocols have been designed for weak adversaries with a restricted view of the system.

This survey concentrates primarily on randomized algorithms, largely because they lie closest to the domain of expertise of the author, but also because they require the least support from the underlying system. Protocols using randomization are discussed starting in Section 3. An excellent survey on work using randomization up to 1989 can be found in [29].

2. Timing assumptions. Consensus can be achieved despite the FLP result by adding timing assumptions to the model that exclude bad executions. Dolev, Dwork, and Stockmeyer [31] characterize the effects of adding limited synchrony assumptions to a message-passing system, and show in particular that consensus becomes possible with up to $n$ faulty processes under a variety of restrictions on the order in which processes take steps or messages are delivered. Dwork, Lynch, and Stockmeyer [34] introduced the partial synchrony model, in which either there is a bound on message delay that is not known to the processes, or there is a known bound that applies only after some initial time $T_{0}$. They describe consensus protocols for this model that work with a bound on the number of faulty processes. Attiya, Dwork, Lynch, and Stockmeyer [16] give a still more refined model in which there are known bounds $C$ on the ratio between the maximum and minimum real time between steps of the same process and $d$ on the maximum message delay; under these assumptions, they prove an upper bound of $(f+1) C d$ and a lower bound of $(f+1) d$ on the amount of real time needed to solve consensus with $f$ faulty processes. Their upper bound uses timeouts to detect failures and can be seen as an early example of the failure detector approach described below.

In the shared-memory framework, a model in which processes can deliberately delay operations in order to avoid overwriting each other's values was used to obtain a very simple and efficient consensus protocol by Alur, Attiya, and Taubenfeld [7]. More recently, Anderson and Moir [8] have used scheduling assumptions from the operating systems world to design protocols that run in constant time when processes run on a uniprocessor under a priority-based scheduling regime or under one that provides minimum scheduling quanta.

3. Failure detectors. With failure detectors, some mechanism exists for notifying other processes that a process has failed. An example 
of a failure detector is the timeout-based mechanism used by Dwork, Lynch, and Stockmeyer [34]. Much more interesting are unreliable failure detectors, where the failure detector can misidentify faulty processes as non-faulty and vice versa. Work on protocols for unreliable failure detectors was initiated by Chandra and Toueg [28]. Chandra, Hadzilacos, and Toueg [27] extended this work by showing the minimum conditions an unreliable failure detector must satisfy to permit consensus. Being able to detect failures, even unreliably, permits solving consensus by electing a coordinator, without the danger of having the protocol hang forever waiting for a failed co-ordinator to wake up. Further examples of work on failure detectors and their limitations in various models can be found in $[5,32]$.

4. Strong primitives. In these models, stronger shared-memory primitives extend or supplement the basic operations of reading and writing registers. Loui and Abu-Amara [44] showed that consensus is solvable with one (but not two) failures using test-and-set bits and is solvable for arbitrarily many failures using three-valued read-modifywrite registers. Extending this work, Herlihy [40] defined a hierarchy of shared-memory objects based on their consensus number, defined somewhat informally as the maximum number of processes for which the object can solve wait-free consensus. The essence of this line of research is that consensus is used as a test problem to prove that certain shared-memory objects cannot implement other, stronger objects. More robust definitions have appeared in subsequent work (see, for example, [42]), and there is now the beginning of a broad theory of the power of shared-memory objects (e.g., [3,48-50]).

Herlihy's paper also gave one of the first universal constructions for arbitrary shared-memory objects based on consensus; showing that objects that can solve consensus with arbitrarily many failures can implement arbitrary shared objects. Another construction is due to Plotkin [46], based on sticky bits similar to the 3-valued read-modifywrite registers of Loui and Abu-Amara [44]. Subsequent constructions have shown how to implement consensus or protocols of equivalent power using primitives such as load-linked/store-conditional [41,52].

Some consensus protocols combine aspects of protocols from different models. For example, a hybrid protocol of Aguilera and Toueg [4] solves consensus very quickly given a failure detector, but solves it eventually using randomization if the failure detector doesn't work. 


\section{Consensus using randomization}

One of the first approaches to solving consensus despite the FLP result was to use randomization. The goal of a randomized consensus protocol is to give the set of non-terminating executions a probability of zero. This does not, in a sense, require breaking the FLP result: these zero-probability executions continue to exist. However, they are irrelevant in practice, provided one is willing to accept a probabilistic termination guarantee. This requires modifying the model as described in Section 4.

The first randomized consensus protocol was given by Ben-Or [20]; as it provides much of the structure for later protocols, we give a description of it in Section 5.1. Later papers extended Ben-Or's work in two directions: the literature on message-passing consensus protocols has largely concentrated on solving agreement problems using cryptographic techniques or private channels with a linear bound on the number of faulty processes (including processes with Byzantine faults, which may misbehave arbitrarily instead of simply stopping); while work in shared-memory systems has used the underlying reliability of the shared-memory system to solve consensus in the wait-free case, where there no limit on how many processes may fail but failures are limited to crash failures. We describe some of the Byzantine agreement work briefly in Section 5.2 and discuss wait-free shared-memory algorithms at greater length in Section 6.

\section{How randomization affects the model}

Adding randomization involves changing both the model, to include the effect of random inputs, and the termination condition, to permit nonterminating executions provided all such executions together have probability zero. ${ }^{1}$

From the point of view of the processes, the main change in the model is the addition of a new COIN-FLIP operation. The COIN-FLIP operation behaves a bit like a READ operation, except that it returns a random value to the process that executes it instead of the value from some register. De-

\footnotetext{
${ }^{1}$ Permitting probability-zero non-terminating executions is not required in a synchronous model, where algorithms exist that use randomization to obtain high efficiency but that still terminate after finitely many rounds in all executions [38,53]. The difference can be accounted for by the fact that deterministic fault-tolerant consensus is possible in a synchronous model. In an asynchronous model, if a randomized consensus protocol terminated in all executions, we could simply replace all coin-flips with hard-wired constants and get a deterministic consensus protocol, contradicting the FLP result.
} 
pending on the precise details of the model, this value might be the outcome of a fair coin-flip, or might be a value chosen from a larger range.

Adding COIN-FLIP operations requires additional changes to the model to handle probabilities of executions. In each state of the system there may be a large number of operations of different processes that may occur next. Previously the choice of which of these operations occurs has been implicit in the choice of a single execution, but now we want to consider ensembles consisting of many executions, where the probabilities of individual executions are determined by the return values of the COIN-FLIP operations. In this framework, it is convenient to assign control of which operation occurs in each configuration to an explicit adversary, a function from partial executions to operations. The idea is that the adversary always chooses what operation happens next, but if that operation is a COIN-FLIP, the result of the operation is random.

The adversary thus takes on the role of the single worst-case execution in the deterministic model. It also takes on the responsibilities of the worstcase execution, in that it must guarantee the fairness conditions required by the algorithm. Once the adversary is specified, which execution occurs is determined completely by the outcome of COIN-FLIP operations, and the probability of an execution or set of executions is determined by the probability of the corresponding coin-flips. We can then talk about the worst-case expected complexity of an algorithm, by considering the worst possible adversary, and then taking the average of some complexity measure over all executions weighted by their probability given this adversary. ${ }^{2}$

Defining the adversary as a function from partial executions to applicable operations means that the adversary can in effect see the entire history of the execution, including outcomes of past coin-flips, internal states of the processes, and the contents of messages and memory locations. Restricting the adversary's knowledge provides an opening for further variations on the model.

The adversary defined above is called the strong adversary. The strong adversary has the advantage of mathematical simplicity, but for practical purposes it may be too strong. While the choice of which operation occurs

\footnotetext{
${ }^{2}$ Technically, it may be that in some models no single worst-case adversary exists; or in other words, that for any adversary, there is a slightly nastier adversary that produces worse performance. An example might be when running under a fairness condition that requires an adversary to eventually permit some operation, but allows different adversaries to delay the operation for arbitrarily long finite times at increasing cost to the algorithm. To handle such possibilities, one can define the worst-case expected complexity instead as the supremum over all adversaries of the expected complexity.
} 
next in a system might depend on the configuration in a complicated way (for example, whether or not reading a particular register causes a page fault might be very difficult to predict without examining the entire previous execution), one can reasonably argue that Nature is not so malicious that only the strong adversary can encompass its awful power. This observation has motivated the development of a variety of weak adversaries that permit faster consensus protocols. ${ }^{3}$ A weak adversary might be unable to break cryptographic tools used by the processes, or it might be restricted more directly by not being allowed to observe anything (such as message or register contents) that would not affect the scheduling mechanism in a real system. Formally, this usually involves restricting the adversary based on a notion of equivalent executions. Two executions are equivalent if they consist of the same sequence of operations (ignoring parameters of operations and their return values), and the adversary must choose the same operation (again ignoring parameters) after any two equivalent partial executions. An adversary that is restricted in this way is called a content-oblivious adversary, since it cannot see the contents of processes, messages, or registers. A still weaker oblivious adversary cannot even distinguish between different operations; it chooses in each state only which process takes the next step. Other models restrict the actions of the adversary, by imposing additional timing assumptions or perturbing the adversary's choices randomly. We describe some of these weak-adversary models in Section 6.1.

In addition to changing the model, we also adapt the correctness conditions for a consensus protocol to reflect the presence of randomness. In particular, the universal quantifier in the termination requirement, which previously spanned all executions, now instead spans all adversaries; and we only require for any particular adversary that the protocol terminate with probability 1.

A similar change in quantification would not be useful for the agreement and validity requirements. Since any violation of agreement or validity must occur after finitely many steps - and thus finitely many coin-flips - any such violation would occur with nonzero probability. Thus we continue to demand that the agreement and validity conditions hold in all executions, which is equivalent to demanding that they hold with probability $1 .{ }^{4}$

\footnotetext{
${ }^{3}$ Conventionally, weak adversaries give rise to strong models, and strong adversaries to weak models; the strength or weakness of the model is a function of how much power it gives to the processes.

${ }^{4}$ It does change the problem to allow disagreement with nonzero probability. A version of the Byzantine agreement problem that permits disagreement but seeks to minimize its probability is studied by Graham and Yao [39]. They cite an earlier unpublished paper of
} 


\section{Fault-tolerant message-passing protocols}

This section describes fault-tolerant message-passing protocols. These achieve consensus given a bound on the number of faults (which may vary from protocol to protocol). We begin with Ben-Or's original exponential-time protocol in Section 5.1 and describe some of its faster descendants in Section 5.2.

\section{$5.1 \quad$ Ben-Or's protocol}

Ben-Or's protocol is the earliest protocol that achieves consensus with probabilistic termination in a model with a strong adversary. Designed for a message-passing system, it tolerates $t<n / 2$ crash failures, and requires exponential expected time to converge in the worst case.

Each process starts off with a preference equal to its input. The protocol proceeds in rounds, each of which has two stages, a voting stage and a ratification stage. Nothing in the system guarantees that all processes proceed through different rounds at the same time; instead, each process keeps track of its own round and uses round labels on messages from other processes to decide whether to use them in its current round, throw them away (for messages from rounds the process has already finished), or save them for later (for messages from rounds the process has not yet reached).

The first stage of each round implements a voting procedure; each process transmits its current preference $p$ to all processes (including itself) by sending a message of the form $(1, r, p)$, and then waits to receive $n-t$ such messages. If any process receives more than $n / 2$ votes for a single value, it causes all processes to decide on this value using the ratification mechanism.

This mechanism is implemented by the second stage of each round. Any process that has observed a majority of votes for $v$ sends a message $(2, r, v$, ratify $)$ to all processes. A process that has not observed a majority for either value sends instead a place-holder message $(2, r, ?)$.

As in the first stage, each process waits to receive at least $n-t$ secondstage messages. Any process that receives even a single $(2, r, v$, ratify) message in round $r$ changes its preference for round $r+1$ to $v$. If, in addition, it receives more than $t$ such messages, it immediately decides on $v$. If, on the other hand, it receives only $(2, r, ?)$ messages, it flips a fair coin to choose a new preference for the next round. The process then continues with the first stage of round $r+1$.

This procedure is summarized as Algorithm 1.

The algorithm guarantees agreement because:

Karlin and Yao as originating this approach. 
Input: boolean value input

Output: boolean value stored in output

Data: boolean preference, integer round

begin

preference $\leftarrow$ input

round $\leftarrow 1$

while true do

send ( 1 , round, preference) to all processes

wait to receive $n-t(1$, round, $*)$ messages

if received more than $n / 2(1$, round, $v)$ messages then

send (2, round, $\mathrm{v}$, ratify) to all processes

else

send $(2$, round, ?) to all processes

end

wait to receive $n-t(2$, round, $*)$ messages

if received a (2, round, $v$, ratify) message then

preference $\leftarrow \mathrm{v}$

if received more than $t$ (2, round, $v$, ratify) messages then

output $\leftarrow \mathrm{v}$

$$
\text { end }
$$

else

preference $\leftarrow$ CoinFlip ()

end

round $\leftarrow$ round +1

end end

Algorithm 1: Ben-Or's consensus protocol. Adapted from [20]. 
1. At most one value can receive a majority of votes in the first stage of a round, so for any two messages $(2, r, v$, ratify $)$ and $\left(2, r, v^{\prime}\right.$, ratify $)$, $v=v^{\prime}$.

2. If some process sees $t+1(2, r, v$, ratify) messages, then every process sees at least one $(2, r, v$, ratify $)$ message.

3. If every process sees a $(2, r, v$, ratify $)$ message, every process votes for $v$ in the first stage of round $r+1$ and every process that has not already decided decides $v$ in round $r+1$.

Validity follows by a similar argument; if all processes vote for the their common input $v$ in round 1 , then all processes send $(2, r, v$, ratify $)$ and decide in the second stage of round 1.

Assuming a weak adversary that cannot observe the contents of messages, termination follows because if no process decides in round $r$, then each process either chooses its new preference based on the majority value $v$ in a $(2, r, v$, ratify $)$ message; or it chooses its new preference randomly, and there is a nonzero probability that all of these random choices equal the unique first-stage majority value (or each other, if there is no majority value). The situation is more complicated with a strong adversary, as different processes may be in the first and second stages at the same time, and so the first-stage majority value may not be determined until after the adversary has seen some of the coin-flips from the second stage. The algorithm continues to work with a strong adversary, but a much more sophisticated proof is needed [6].

Unfortunately, in either case the probability that the algorithm terminates in any given round may be exponentially small as a function of the number of processes, requiring exponentially many rounds. Note also that each process continues to run the protocol even after deciding; however, the protocol can be modified so that each process exits at most one round after first setting its output value.

\section{$5.2 \quad$ Faster protocols}

Ben-Or's protocol not only showed that consensus becomes possible with randomization, but also initiated a large body of work on randomized protocols for the harder problem of Byzantine agreement, in which faulty processes can exhibit arbitrary behavior instead of simply crashing. This work has generally assumed the availability of cryptographic tools. Rabin [47] showed that Byzantine agreement can be solved in constant expected time 
given a shared coin visible to all processes, and described an implementation of such a coin based on digital signatures and a trusted dealer. Feldman and Micali [35] gave a constant-round shared-coin for a synchronous system that uses secret sharing to avoid the need for a trusted dealer.

A constant-time shared coin for an asynchronous system was given by Canetti and Rabin [25] based in part on further unpublished work by Feldman. For the Canetti and Rabin protocol the cryptographic assumptions can be replaced by the assumption of private channels. A simplified presentation of the Canetti-Rabin protocol that tolerates crash failures only is given in [17, Section 14.3.2].

\section{Wait-free shared-memory protocols}

A protocol that tolerates up to $n-1$ crash failures is called wait-free, because it means that any process can finish the protocol in isolation without waiting for the others. Wait-free message-passing protocols for all but trivial problems are easily shown to be impossible, as a process running in isolation cannot tell whether it is the sole survivor or simply the victim of a network partition [23].

With shared memory, things become easier. Even though processes may fail, it is usually assumed that data in the shared memory survives. ${ }^{5}$ This eliminates the possibility of partition - even a process running in isolation can still read the notes left behind by dead processes that ran before. The consensus problem then becomes a problem of getting the contents of the shared memory into a state that unambiguously determines the decision value, even for processes that may have slept through most of the protocol.

In this section, we begin describing the history of wait-free sharedmemory consensus, and then give examples of different approaches to the problem, showing the range of the trade-off between efficiency and robustness against adversaries of increasing strength.

The first randomized protocol to use shared memory to solve consensus was described by Chor, Israeli, and Li [30], for a model in which processes can generate random values and write them to shared memory in a single atomic operation (this is equivalent to assuming a weak adversary that cannot see internal coin-flips until they are written out). A noteworthy feature of the protocol is that it works for values in any input range, not just binary values.

We describe the Chor-Israeli-Li protocol in more detail in Section 6.1.1. To give a brief summary, the essential idea of the Chor-Israeli-Li protocol

\footnotetext{
${ }^{5}$ See $[2,43]$ for models in which the shared memory itself can be faulty.
} 
is to have the processes run a race, where processes advance through a sequence of rounds as in the Ben-Or protocol, and slow processes adopt the preferences of faster processes that have already reached later rounds. If a single process eventually pulls far enough ahead of all processes that disagree with it, both this leader and the other processes can safely decide on the leader's preference, knowing that any other processes will adopt the same preference by the time they catch up. Processes flip coins to decide whether or not to advance to the next level. With the probability of advancement set at $\frac{1}{2 n}$, a leader emerges after $O\left(n^{2}\right)$ total work.

With a strong adversary, the Chor-Israeli-Li protocol fails. The winning strategy for the adversary is to construct a "lockstep execution" that keeps all processes at the same round, by stopping any process that has incremented its round until all other processes have done so as well. This strategy necessarily requires that the adversary be able to observe internal states of the processes when making its scheduling decisions. It is still possible to solve consensus with a strong adversary, but a different approach is needed.

Wait-free consensus protocols that tolerate a strong adversary began to appear soon after the publication of the conference version of the ChorIsraeli-Li paper. Abrahamson [1] gave the first randomized wait-free consensus protocol for a strong adversary. Though described in terms of processes acquiring locks of increasing strengths, if the strengths of the locks are interpreted as round numbers it superficially resembles the Ben-Or protocol translated to shared memory. As in Ben-Or's protocol, the method for obtaining agreement is to have the processes choose new preferences at random; after $2^{O\left(n^{2}\right)}$ steps on average, the processes' random choices will have agreed for a long enough period that the adversary cannot manipulate them into further disagreement.

Also as with Ben-Or's protocol, eliminating exponential waiting time required replacing the independent local coins of the processes with global coin protocols shared between the processes. The first protocol to do this was given by Aspnes and Herlihy [12]; it used a round structure similar to the Chor-Israeli-Li protocol but relied on a shared coin protocol based on majority voting to shake the processes into agreement. The Aspnes and Herlihy protocol was still fairly expensive, requiring an expected $O\left(n^{4}\right)$ total operations in the worst case, though much of this cost was accounted for by the overhead of using a primitive $O\left(n^{2}\right)$-work snapshot subroutine constructed specifically for the protocol. Subsequent work with the strong adversary by many authors $[9,14,15,21,22,33,51]$ has largely retained the overall structure of the Aspnes and Herlihy protocol, while reducing the overhead and elimi- 
nating in some cases annoyances like the use of unboundedly large registers. The net result of these developments was to reduce the expected total work to achieve consensus to $O\left(n^{2} \log n\right)$ using the shared-coin protocol of Bracha and Rachman [22] and the expected per-process work to $O\left(n \log ^{2} n\right)$ using the shared-coin protocol of [14]. An $O\left(n^{2} \log n\right)$ consensus protocol using the Bracha-Rachman coin is described in Section 6.2.

Further improvements in the strong-adversary model stalled at this point. It was later shown by Aspnes [10] that no strong-adversary consensus protocol could run in less than $\Omega\left(n^{2} / \log ^{2} n\right)$ total work in essentially any model in which the FLP bound applies. The essential idea of this lower bound was to extend the classification of states in the FLP argument as bivalent or univalent to a randomized framework, by defining a state as bivalent if the adversary can force either decision value to occur with high probability, univalent if it can force only one decision value to occur with high probability, and null-valent if it can force neither decision value to occur with high probability. This last case is equivalent to saying that the adversary cannot bias the outcome of the protocol too much - or, in other words, that the protocol acts like a shared coin. Aspnes showed, using a variant of the FLP argument, that any consensus protocol that reaches a decision in less than $n^{2}$ steps is likely to pass through a null-valent state, and provided a separate lower bound on shared coins to show that $\Omega\left(n^{2} / \log ^{2} n\right)$ expected work (specifically, $\Omega\left(n^{2} / \log ^{2} n\right)$ expected write operations) would be needed to finish any protocol starting in a null-valent state. Combining these two results gives the lower bound on consensus.

Even before this lower bound was known, the lack of further improvement in strong-adversary protocols led to greater interest in protocols for weak adversaries. Aumann and Bender [19] gave a shared coin algorithm for the value-oblivious adversary that cannot observe the internal states of the processes or values that have been written to memory but not yet read. Based on propagating values through a butterfly network, their algorithm gives a constant-bias shared coin in $O\left(n \log ^{2} n\right)$ total work. Concurrently, Chandra devised an algorithm for repeatedly solving consensus in a model with essentially the same adversary, with a polylogarithmic per-process time bound. Chandra's protocol uses a stockpile of pre-flipped coins that the processes agree to use. The initial execution of the protocol is expensive, due to the need to generate an initial stockpile of unused coins, but subsequent executions can solve new instances of the consensus problem in only $O\left(\log ^{2} n\right)$ time. Chandra's algorithm also gives a very streamlined implementation of the rounds mechanism from earlier strong-adversary protocols, reducing the shared data needed to just two arrays of multi-writer bits. (We use a ver- 
sion of this algorithm to reduce consensus to shared coin in Section 6.2.1.) Soon afterwards, Aumann [18] showed how to achieve $O(\log n)$ expected perprocess work even for a single iteration of consensus. It is not clear whether $O(\log n)$ expected steps is the best possible in this model, or whether further improvements may be obtained.

\subsection{Weak-adversary protocols}

In this section, we first describe the Chor-Israeli-Li protocol that demonstrated the possibility of wait-free consensus, and then sketch out some more recent work that uses a similar approach. The unifying theme of these protocols is to have the processes run a race where advancement to the next phase is controlled by some random process and winning the race (by getting far enough ahead of the other processes) determines the outcome of the protocol. Although the adversary can use its control over timing to handicap particular processes, a weak adversary cannot identify which phase each process is in and thus cannot prevent a victor from emerging.

\subsubsection{The Chor-Israeli-Li protocol}

Pseudocode for a simplified version of the Chor-Israeli-Li protocol is given as Algorithm 2.

Communication between processes is done by having each process alternate between writing out its current round and preference to its own output register in Line 1, and reading all the other processes' registers to observe their recent states in Line 2. The only interactions with the shared memory are in these two lines. A process notices that the race has been won if it observes that processes with preference $v$ are far enough ahead of all disagreeing processes in Line 3 ; in this case, it decides on $v$ and exits. If the process does not decide, it adopts the common preference of the fastest processes provided they all agree (Lines $4-5$ ).

The only tricky part is ensuring that eventually some process does win the race, i.e. moves far enough ahead of any processes that disagree with it. This is done in Line 6 by having each process choose whether to advance to the next round at random. Chor et al. show that, provided the adversary cannot delay a process's write depending on its choice to advance or not, a leader emerges on average after $O(n)$ passes through the loop. The expected total work is $O\left(n^{2}\right)$, since each pass requires $O(n)$ read operations. 


\section{Input: input (an arbitrary value)}

Output: return value

Local data: preference, round, maxround

Shared data: one single-writer multi-reader register for each pro-

\section{begin} cess

preference $\leftarrow$ input

round $\leftarrow 1$

while true do

$1 \quad$ write (preference, round)

2 read all registers $R$

$$
\text { maxround } \leftarrow \max _{R} R \text {.round }
$$

$3 \quad$ if for all $R$ where $R$.round $\geq$ maxround -1 , R.preference $=v$ then return $v$

$$
\text { else }
$$

$4 \quad$ if exists $v$ such that for all $R$ where R.round = maxround, R.preference $=v$ then

$5 \quad$ preference $\leftarrow v$

$6 \quad$ end $\quad$ with probability $\frac{1}{2 n}$ do

round $\leftarrow \max ($ round +1 , maxround -2$)$

end end end

Algorithm 2: Chor-Israeli-Li protocol. Adapted with modifications from [30]. 


\subsubsection{Protocols for still weaker adversaries}

For still weaker adversaries, it is possible to remove the randomization from the Chor-Israeli-Li protocol and still solve consensus. This is essentially what is done in the uniprocessor consensus protocol of Anderson and Moir [8], which relies on quantum and/or priority-based scheduling to avoid lockstep executions and which achieves consensus deterministically in constant work per process; and in the "noisy environment" consensus protocol of Aspnes [11], which assumes that the adversary's schedule is perturbed by cumulative random noise and achieves consensus in expected $O(\log n)$ work per process.

\subsection{Strong-adversary protocols}

The main tool for defeating a strong adversary - one that can react to the internal states of processes and the contents of memory - has been the use of weak shared coin protocols. These provide a weak shared coin abstraction with the property that, regardless of the adversary's behavior, for each value 0 or 1 there is a constant minimum probability $\epsilon$ that all processes agree on that value as the value of the shared coin. The coin is said to be weak because $\epsilon$ is in general less than $\frac{1}{2}$ : there will be some executions in which the adversary either seizes control of the coin or prevents agreement altogether.

We describe a typical weak shared coin protocol in Section 6.2.2. As in most such protocols, the shared coin is obtained by taking a majority of many local coin flips generated by individual processes. While the adversary can bias the outcome of the coin by selectively killing processes that are planning to vote the wrong way, it can only hide up to $n-1$ votes in this way, and with enough votes it is likely that the majority value will not shift as a result of the adversary's interference.

Before presenting a shared coin protocol, we will show that having such a protocol does indeed give a solution to consensus.

\subsubsection{Consensus from a shared coin}

Given a polynomial-work shared coin protocol, it is easy to build a wait-free shared-memory consensus protocol requiring similar total work. The basic idea is the same as in the Ben-Or protocol: disagreements are eliminated by sending all but the fastest processes off to flip coins. The actual structure of the algorithm resembles the Chor-Israeli-Li algorithm, in that processes proceed through a sequence of rounds, and slow processes adopt the preferences of faster ones, but now the rounds structure is no longer used to distinguish 
winners from losers but instead simply ensures that the fastest processes do not have to wait for processes stuck in earlier rounds. With appropriate machinery, we can arrange that in each round all processes either (a) think they are leaders and agree with all other leaders, or (b) participate in the shared coin protocol. Since all processes in the first category agree with each other, and all processes in the second category will also choose this agreed-upon value with probability at least $\epsilon$, after $O(1 / \epsilon)=O(1)$ rounds all processes agree.

The first wait-free consensus protocol to use this technique was the protocol of Aspnes and Herlihy [12], which included an ad-hoc snapshot algorithm and various other additional mechanisms to allow the protocol to be built from single-writer registers using the techniques of the day. A more recent algorithm due to Chandra [26] gives a much simpler implementation of the multi-round framework using two arrays of multi-writer bits.

Pseudocode for a simplified version of this algorithm is given as Algorithm 3. The main simplification the use of unbounded bit-vectors, which avoids some additional machinery in Chandra's algorithm for truncating the protocol if it has not terminated in $O(\log n)$ rounds and switching to a slower bounded-space algorithm. ${ }^{6}$ The simplified algorithm requires very few assumptions about the system. In particular, it works even when processes do not have identities [24] and with infinitely many processes [13].

We will not give a detailed proof of correctness of Algorithm 3, referring the interested reader instead to [26]. However, we can give some intuition about why it works.

The two arrays of bits substitute for the round fields in the Chor-Israeli$\mathrm{Li}$ algorithm, allowing a process to quickly determine if its preference is in the lead without having to read $n$ separate registers. A process $P$ that reaches round $r$ with preference $p$ registers this fact by setting $\operatorname{mark}[p][r]$ in Line 1.

What $P$ does next depends on whether it sees itself behind, tied with, one round ahead, or two rounds ahead of the fastest process with the opposite preference:

- If it sees a mark in mark $[1-p][r+1]$, it knows that it is behind, and

\footnotetext{
${ }^{6}$ This trick, of switching from a fast algorithm that is running too long to a slower but bounded algorithm was originally devised by Goldreich and Petrank [38] for synchronous Byzantine agreement protocols. Chandra's original algorithm was designed for a weak adversary and its shared coin subroutine consumes "pre-flipped" shared coins stored in memory. Switching to a second algorithm is needed to avoid running out of these preflipped coins. But since the switch occurs with low probability, the cost of the slower algorithm is rarely incurred, and thus does not change the total asymptotic expected cost.
} 
Input: boolean value input

Local data: boolean preference $p$, integer round $r$, boolean new preference $p^{\prime}$

Shared data: boolean $\operatorname{mark}[b][i]$ for each $b \in\{0,1\}, i \in Z^{+}$, of which mark[0][0] and mark[1][0] are initialized to true while all other elements are initialized to false.

Subprotocols: Shared coin protocols SharedCoin $\mathrm{n}_{r}$ for $r=0,1, \ldots$ begin

$p \leftarrow$ input

$r \leftarrow 1$

while true do

$1 \quad \operatorname{mark}[p][r] \leftarrow$ true

$$
\text { if } \operatorname{mark}[1-p][r+1] \text { then }
$$

$2 \quad p^{\prime} \leftarrow 1-p$

else if $\operatorname{mark}[1-p][r]$ then

$3 \quad p^{\prime} \leftarrow$ SharedCoin $_{r}()$

else if $\operatorname{mark}[1-p][r-1]$ then

$4 \quad p^{\prime} \leftarrow p$

else

$5 \quad$ return $p$

end

if $\operatorname{mark}[p][r+1]=$ false then

$6 \quad p \leftarrow p^{\prime}$

$$
\text { end }
$$

7 end

$r \leftarrow r+1$

Algorithm 3: Wait-free consensus using shared coins. Adapted with modifications from [26], Figure 1. 
switches to the other preference (Line 2).

- If the latest mark it sees is in mark $[1-p][r]$, it assumes it is tied, and chooses a new preference using the shared coin (Line 3 ).

- If the latest mark it sees is in mark $[1-p][r-1]$, it keeps its current preference (Line 4) but does not yet decide, as it may be that some process $Q$ with the opposite preference is close enough that $Q$ will immediately set mark $[1-p][r]$ and then think it is tied with $P$.

- Finally, if it sees no mark later than mark $[1-p][r-2]$ (which it doesn't bother to read), then any $Q$ with a different preference has not yet executed Line 1 in round $r-1$; and so after $Q$ has done so, it will see the mark that $P$ already put in $\operatorname{mark}[p][r]$, and then switch its preference to $P$ 's preference $p$. In this case $P$ can safely decide $p$ (Line 5 ) knowing that any slower process will switch to the same preference by the time it catches up.

As a last step, the process checks to see if at least one process with its old preference has already advanced to the next round. In this case, it discards its decisions from the current round and sticks with its previous preference; otherwise, it adopts the new preference it determined in the preceding lines (Line 6). This avoids a problem where some process $P$ decides $p$ in $r$, but a process $P^{\prime}$ with the same preference later sees a tie in $r-1$ (after some other processes catch up with it), executes the shared coin, and suddenly switches its preference while catching up with $P$.

Intuitively, agreement holds precisely because of the explanations for Lines 5 and 6 . But a full correctness proof is subtle, and depends among other things on the precise order in which mark $[1-p][r+1]$, mark $[1-p][r]$, and mark $[1-p][r-1]$ are read in Algorithm 3. However, if one accepts that the algorithm satisfies agreement, it is trivial to see that it also satisfies validity, because no process every changes its preference unless it first sees that some other process has a different preferences.

Termination follows from the properties of the shared coin. Note that it is possible that some processes tied for the lead will skip the coin because they read mark $[1-p][r]$ just before the others write it. But it is not hard to show that these processes will at least agree with each other. So the processes that do participate in the coin will fall into agreement with the non-participants with at least a constant probability, due to the bounded bias of the coin, and agreement is reached after a constant number of rounds on average. Since the overhead of the consensus protocol is small (five operations per 
round in the worst case), the cost is dominated by the cost of the shared coin protocol.

\subsubsection{Bracha and Rachman's shared coin protocol}

We can use any shared coin subroutine we like in Chandra's protocol; as discussed previously, the expected cost of the algorithm will be within a constant factor of the cost of the shared coin, provided the shared coin guarantees at most constant bias. The most efficient shared coin protocol currently known for the strong adversary, when measured according to expected total work, is Bracha and Rachman's 1991 voting protocol [22]. Pseudocode for this protocol is given as Algorithm 4.

The intuition behind this protocol is the same as for all voting-based protocols: The processes collectively generate many "common votes," which in this case consist of all votes generated before $n^{2}$ votes have been written to the registers. Each process's view of the common votes is obscured both by additional "extra votes" that are generated by processes that have not yet noticed that there are enough votes in the registers, and by the adversary's selective removal of "hidden votes" by delaying processes between generating votes in Line 1 and writing them out in Line $2 .{ }^{7}$ The reason the protocol works is that we can argue that the $n^{2}$ common votes have at least a constant probability of giving a majority large enough that neither the random drift of up to $n^{2} / \log n$ extra votes nor the selective pressure of up to $n-1$ hidden votes is likely to change the apparent outcome.

Because the extra votes are not biased by the adversary, they are less dangerous than the hidden votes and we can tolerate more of them. This is why the protocol can amortize the cost of the $n$ read operations to detect termination in Line 3 over the $\frac{n}{\log n}$ votes generated in the inside loop. This amortized termination test was the main contribution of the Bracha and Rachman protocol, and was what finally brought the expected total work for consensus down to nearly $O\left(n^{2}\right)$ from the $O\left(n^{3}\right)$ (or worse) bounds on previous protocols.

In detail, the votes are classified as follows. These classes are not exclusive; a vote that is in either of the first two classes may also be in the last.

\footnotetext{
${ }^{7}$ Our explanation of the Bracha-Rachman protocol follows the analysis of a similar protocol from [14]. Bracha and Rachman's original analysis in [22] uses a slightly different classification that includes common and extra votes but does not separate out the issue of hidden votes. Their classification requires the analysis of a more sophisticated random process than the one considered here.
} 
Input: none

Output: boolean output

Local data: boolean preference $p$; integer round $r$; utility variables $c$, total, and ones

Shared data: single-writer register $r[p]$ for each process $p$, each of which holds a pair of integers (flips, ones), initially $(0,0)$

\section{begin}

repeat

$$
\begin{array}{ll} 
& \text { for } i \leftarrow 1 \text { to } \frac{n}{\log n} \text { do } \\
1 & c \leftarrow \text { Coinflip }() \\
2 & r[p] \leftarrow(r[p] . \text { flips }+1, r[p] . \text { ones }+c)
\end{array}
$$

$1 \quad c \leftarrow$ Coinflip ()

$3 \quad$ Read all registers $r[p]$

$$
\text { total } \leftarrow \sum_{p} r[p] \text {.flips }
$$

until total $>n^{2}$

$4 \quad$ Read all registers $r[p]$

total $\leftarrow \sum_{p} r[p]$.flips

ones $\leftarrow \sum_{p} r[p]$.ones

$5 \quad$ if $\frac{\text { total }}{\text { ones }} \geq \frac{1}{2}$ then

return 1

else

return 0

end

end

Algorithm 4: Bracha and Rachman's voting protocol. Adapted from [22], Figure 2. 
1. Common votes consist of all votes generated before the sum of the $r[p]$.total fields exceeds $n^{2}$. In a sense, these are all the votes that would be seen by all processes if they had been written out immediately. There will be between $n^{2}+1$ and $n^{2}+n$ such votes.

2. Extra votes for process $P$ are those votes $X_{i}$ that are not part of the common votes and that are generated by some process $Q$ before $P$ reads $r[Q]$ in Line 4 . Each process $Q$ contributes at most $\frac{n}{\log n}$ such extra votes, because it cannot generate more without executing the termination test staring in Line 3 . The common votes plus the extra votes for $P$ include all votes that $P$ would have seen had they been written out immediately.

3. Hidden votes for $P$ are those votes which were generated by some process $Q$ but not written to $r[Q]$ when $P$ reads $r[Q]$. Each process $Q$ contributes at most one hidden vote for $P$.

The total vote for $P$ is given by:

$($ common votes $)+($ extra votes for $P)-($ hidden votes for $P)$.

When the adversary permits a process to flip its coin in Line 1, it is already determined whether or not that coin-flip will count towards the common votes or the extra votes for any particular process $P$. So both the common votes and the extra votes consist of a sequence of unbiased fair coins, and the only power the adversary has over them is the choice of when to stop the sequence.

Using the normal approximation to the binomial distribution, it is possible to show that the net majority for 1 of the approximately $n^{2}$ common votes is at least $3 n$ with some constant probability $p$. Adding in the $\frac{n^{2}}{\log n}$ extra votes for a particular process $P$ may adjust this total up or down; it reduces it below $n$ only if at some point during an unbiased random walk of length $\frac{n^{2}}{\log n}$ the total drops below $-2 n$. Standard results 8 show that this probability is bounded by $\frac{1}{n^{2}}$, so even when we multiply the probability for a single process by the number of processes $n$, the probability that the extra votes are less than $n$ for any $P$ is still less than $\frac{1}{n}$. We thus have a probability of at least $p\left(1-\frac{1}{n}\right)$ that the common votes plus the extra votes for all $P$ are at least $n$. Since each $P$ has at most $n-1$ hidden votes, each $P$ then sees a positive net vote and decides 1 .

${ }^{8}$ E.g. [36, Theorem III.7.3]. 
The preceding argument shows that when $n>1$, all processes decide 1 with at least a constant probability $\epsilon=p / 2$. The case for decision value 0 is symmetric.

The total work of Algorithm 4 is $O\left(n^{2} \log n\right)$; there are $O\left(n^{2}\right)$ votes cast by all of the processes together, and each vote has an amortized cost of $O(\log n)$. Plugging the Bracha-Rachman shared coin into Algorithm 3 thus gives a consensus protocol whose expected total work is also $O\left(n^{2} \log n\right)$.

This is the current best known bound on expected total work for waitfree consensus in the strong-adversary model. Since the Bracha-Rachman algorithm, the only further improvement in this model has been the AspnesWaarts shared coin [14], which modifies the Bracha-Rachman coin to prevent any single process from having to perform more than $O\left(n \log ^{2} n\right)$ work, at the cost of increasing the total work bound to $O\left(n^{2} \log ^{2} n\right)$.

There is still some room left to improve the total work, but not much. We have previously mentioned the $\Omega\left(n^{2} / \log ^{2} n\right)$ lower bound on the expected number of write operations for any wait-free consensus protocol from [10]. The same paper conjectured that the actual lower bound is $\Omega\left(n^{2} / \log n\right)$. Using this conjectured lower bound, and throwing in an extra logarithmic factor for the cost of amortizing reads over coin-flips and writes, a reasonable guess for the true cost of consensus in this model might be $\Theta\left(n^{2}\right)$.

\section{Acknowledgments}

The author would like to thank Prasad Jayanti and Sam Toueg for many useful comments on the original abstract and outline of this survey, the students in Yale's Fall 2001 Theory of Distributed Systems class for their patience with an early draft of some of the later sections, Hagit Attiya and Sergio Rajsbaum for suggesting some references, the anonymous referees for their extensive and useful comments, and Marcos Aguilera for identifying and providing fixes for several serious technical errors.

\section{References}

[1] Karl R. Abrahamson. On achieving consensus using a shared memory. In Proceedings of the Seventh Annual ACM Symposium on Principles of Distributed Computing, pages 291-302, Toronto, Ontario, Canada, August 1988. 
[2] Y. Afek, D.S. Greenberg, M. Merritt, and G. Taubenfeld. Computing with faulty shared objects. Journal of the Association of the Computing Machinery, 42:1231-1274, 1995.

[3] Yehuda Afek, Michael Merritt, and Gadi Taubenfeld. The power of multiobjects. Information and Computation, 153(1):117-138, 1999.

[4] Aguilera and Toueg. Failure detection and randomization: A hybrid approach to solve consensus. SIAM Journal on Computing, 28(3):890903, 1998.

[5] M. K. Aguilera, W. Chen, and S. Toueg. Failure detection and consensus in the crash recovery model. Distributed Computing, 13(2):99-125, April 2000.

[6] Marcos Kawazoe Aguilera and Sam Toueg. Correctness proof of benor's randomized consensus algorithm. Technical Report TR98-1682, Cornell University, Computer Science, May 17, 1998.

[7] Rajeev Alur, Hagit Attiya, and Gadi Taubenfeld. Time-adaptive algorithms for syncronization. SIAM Journal on Computing, 26(2):539-556, April 1997.

[8] James H. Anderson and Mark Moir. Wait-free syncronization in multiprogrammed systems: Integrating priority-based and quantum-based scheduling. In Proceedings of the Eighteenth Annual ACM Symposium on Principles of Distributed Computing, pages 123-132, Atlanta, Georgia, USA, 3-6 May 1999.

[9] James Aspnes. Time- and space-efficient randomized consensus. Journal of Algorithms, 14(3):414-431, May 1993.

[10] James Aspnes. Lower bounds for distributed coin-flipping and randomized consensus. Journal of the ACM, 45(3):415-450, May 1998.

[11] James Aspnes. Fast deterministic consensus in a noisy environment. In Proceedings of the Nineteenth Annual ACM Symposium on Principles of Distributed Computing, pages 299-309, Portland, Oregon, July 2000. To appear, Journal of Algorithms.

[12] James Aspnes and Maurice Herlihy. Fast randomized consensus using shared memory. Journal of Algorithms, 11(3):441-461, September 1990. 
[13] James Aspnes, Gauri Shah, and Jatin Shah. Wait-free consensus with infinite arrivals. In Proceedings of the 34th Annual ACM Symposium on the Theory of Computing, pages 524-533, 19-21 May 2002.

[14] James Aspnes and Orli Waarts. Randomized consensus in expected $O\left(N \log ^{2} N\right)$ operations per processor. SIAM Journal on Computing, 25(5):1024-1044, October 1996.

[15] Hagit Attiya, Danny Dolev, and Nir Shavit. Bounded polynomial randomized consensus. In Proceedings of the Eighth Annual ACM Symposium on Principles of Distributed Computing, pages 281-293, Edmonton, Alberta, Canada, 14-16 August 1989.

[16] Hagit Attiya, Cynthia Dwork, Nancy Lynch, and Larry Stockmeyer. Bounds on the time to reach agreement in the presence of timing uncertainty. Journal of the ACM, 41(1):122-152, January 1994.

[17] Hagit Attiya and Jennifer Welch. Distributed Computing: Fundamentals, Simulations, and Advanced Topics. McGraw-Hill, 1998.

[18] Yonatan Aumann. Efficient asynchronous consensus with the weak adversary scheduler. In Proceedings of the Sixteenth Annual ACM Symposium on Principles of Distributed Computing, pages 209-218, Santa Barbara, California, 21-24 August 1997.

[19] Yonatan Aumann and Michael A. Bender. Efficient asynchronous consensus with the value-oblivious adversary scheduler. In Friedhelm Meyer auf der Heide and Burkhard Monien, editors, Automata, Languages and Programming, 23rd International Colloquium, volume 1099 of Lecture Notes in Computer Science, pages 622-633, Paderborn, Germany, 8-12 July 1996. Springer-Verlag.

[20] Michael Ben-Or. Another advantage of free choice: Completely asynchronous agreement protocols (extended abstract). In Proceedings of the Second Annual ACM SIGACT-SIGOPS Symposium on Principles of Distributed Computing, pages 27-30, Montreal, Quebec, Canada, August 1983.

[21] Gabriel Bracha and Ophir Rachman. Approximated counters and randomized consensus. Technical Report 662, Technion, 1990.

[22] Gabriel Bracha and Ophir Rachman. Randomized consensus in expected $O\left(n^{2} \log n\right)$ operations. In Sam Toueg, Paul G. Spirakis, and 
Lefteris M. Kirousis, editors, Distributed Algorithms, 5th International Workshop, volume 579 of Lecture Notes in Computer Science, pages 143-150, Delphi, Greece, 7-9 October 1991. Springer, 1992.

[23] Gabriel Bracha and Sam Toueg. Asynchronous consensus and broadcast protocols. Journal of the ACM, 32(4):824-840, October 1985.

[24] Harry Buhrman, Alessandro Panconesi, Riccardo Silvestri, and Paul M. B. Vitanyi. On the importance of having an identity or is consensus really universal? In Proceedings of the Fourteenth International Symposium on Distributed Computing, pages 134-148, Toledo, Spain, 4-6 October 2000.

[25] Ran Canetti and Tal Rabin. Fast asynchronous Byzantine agreement with optimal resilience (extended abstract). In Proceedings of the Twenty-Fifth Annual ACM Symposium on the Theory of Computing, pages 42-51, San Diego, California, 16-18 May 1993.

[26] Tushar Deepak Chandra. Polylog randomized wait-free consensus. In Proceedings of the Fifteenth Annual ACM Symposium on Principles of Distributed Computing, pages 166-175, Philadelphia, Pennsylvania, USA, 23-26 May 1996.

[27] Tushar Deepak Chandra, Vassos Hadzilacos, and Sam Toueg. The weakest failure detector for solving Consensus. Journal of the ACM, 43(4):685-722, July 1996.

[28] Tushar Deepak Chandra and Sam Toueg. Unreliable failure detectors for reliable distributed systems. Journal of the ACM, 43(2):225-267, March 1996.

[29] B. Chor and C. Dwork. Randomization in Byzantine agreement. In Advances in Computing Research 5: Randomness and Computation, pages 443-497. JAI Press, 1989.

[30] Benny Chor, Amos Israeli, and Ming Li. Wait-free consensus using asynchronous hardware. SIAM Journal on Computing, 23(4):701-712, August 1994.

[31] Danny Dolev, Cynthia Dwork, and Larry Stockmeyer. On the minimal synchronism needed for distributed consensus. Journal of the ACM, 34(1):77-97, January 1987. 
[32] Danny Dolev, Roy Friedman, Idit Keidar, and Dahlia Malkhi. Failure detectors in omission failure environments. In Proceedings of the Sixteenth Annual ACM Symposium on Principles of Distributed Computing, page 286, 1997.

[33] Cynthia Dwork, Maurice Herlihy, Serge Plotkin, and Orli Waarts. Time-lapse snapshots. SIAM Journal on Computing, 28(5):1848-1874, 1999.

[34] Cynthia Dwork, Nancy Lynch, and Larry Stockmeyer. Consensus in the presence of partial synchrony. Journal of the ACM, 35(2):288-323, April 1988.

[35] Pesech Feldman and Silvio Micali. An optimal probabilistic protocol for synchronous Byzantine agreement. SIAM Journal on Computing, 26(4):873-933, August 1997.

[36] William Feller. An Introduction to Probability Theory and Its Application. Wiley, 3rd edition, 1970. (Revised printing).

[37] Michael J. Fischer, Nancy A. Lynch, and Michael S. Paterson. Impossibility of distributed consensus with one faulty process. Journal of the ACM, 32(2):374-382, April 1985.

[38] Oded Goldreich and Erez Petrank. The best of both worlds: Guaranteeing termination in fast randomized byzantine agreement protocols. Information Processing Letters, 36(1):45-49, 1990.

[39] Ronald L. Graham and Andrew C. Yao. On the improbability of reaching Byzantine agreement. In Proceedings of the 21st Annual ACM Symposium on the Theory of Computing, pages 467-478, 15-17 May 1989.

[40] Maurice Herlihy. Wait-free synchronization. ACM Transactions on Programming Languages and Systems, 13(1):124-149, January 1991.

[41] Maurice Herlihy. A methodology for implementing highly concurrent data objects. ACM Transactions on Programming Languages and Systems, 15(5):745-770, November 1993.

[42] Prasad Jayanti. Robust wait-free hierarchies. Journal of the ACM, 44(4):592-614, July 1997.

[43] Prasad Jayanti, Tushar Deepak Chandra, and Sam Toueg. Faulttolerant wait-free shared objects. Journal of the ACM, 45(3):451-500, May 1998. 
[44] Michael C. Loui and Hosame H. Abu-Amara. Memory requirements for agreement among unreliable asynchronous processes. In Franco P. Preparata, editor, Advances in Computing Research, volume 4. JAI Press, 1987.

[45] M. Pease, R. Shostak, and L. Lamport. Reaching agreements in the presence of faults. Journal of the ACM, 27(2):228-234, April 1980.

[46] S. A. Plotkin. Sticky bits and universitality of consensus. In Proceedings of the Eighth Annual ACM Symposium on Principles of Distributed Computing, pages 159-176, New York, NY, 1989. ACM Press.

[47] M. O. Rabin. Randomized Byzantine Generals. In Proc. 24th Ann. IEEE Symp. on Foundations of Computer Science, pages 403-409, 1983.

[48] Eric Ruppert. Determining consensus numbers. In Proceedings of the Sixteenth Annual ACM Symposium on Principles of Distributed Computing, pages 93-99, 1997.

[49] Eric Ruppert. Consensus numbers of multi-objects. In Proceedings of the Seventeenth Annual ACM Symposium on Principles of Distributed Computing, pages 211-217, 1998.

[50] Eric Ruppert. Consensus numbers of transactional objects. In International Symposium on Distributed Computing, pages 312-326, 1999.

[51] Michael Saks, Nir Shavit, and Heather Woll. Optimal time randomized consensus - making resilient algorithms fast in practice. In Proceedings of the Second Annual ACM-SIAM Symposium on Discrete Algorithms, pages 351-362, San Francisco, California, 28-30 January 1991.

[52] Nir Shavit and Dan Touitou. Software transactional memory. In Proceedings of the Fourteenth Annual ACM Symposium on Principles of Distributed Computing, pages 204-213, 1995.

[53] Arkady Zamsky. A randomized Byzantine agreement protocol with constant expected time and guaranteed termination in optimal (deterministic) time. In Proceedings of the Fifteenth Annual ACM Symposium on Principles of Distributed Computing, pages 201-208, Philadelphia, Pennsylvania, USA, 23-26 May 1996. 